\title{
Association between hypo- and hyperkalemia and outcome in acute heart failure patients : the role of medications
}

\section{GREAT Global Res Acute Conditions}

2018-03

GREAT Global Res Acute Conditions , INI-CRCT Invest Network Initiative , Legrand , M , Lassus, J \& Harjola , V-P 2018 , ' Association between hypo- and hyperkalemia and outcome in acute heart failure patients : the role of medications ' , Clinical Research in Cardiology , vol. 107 , no. 3 , pp. 214-221 . https://doi.org/10.1007/s00392-017-1173-3

http://hdl.handle.net/10138/300885

https://doi.org/10.1007/s00392-017-1173-3

publishedVersion

Downloaded from Helda, University of Helsinki institutional repository.

This is an electronic reprint of the original article.

This reprint may differ from the original in pagination and typographic detail.

Please cite the original version. 


\title{
Association between hypo- and hyperkalemia and outcome in acute heart failure patients: the role of medications
}

\author{
Matthieu Legrand ${ }^{1,2,3,4} \oplus$ - Pierre-Olivier Ludes ${ }^{1,2} \cdot$ Ziad Massy $^{5,6} \cdot$ Patrick Rossignol $^{7} \cdot$ Jiri Parenica $^{8,9}$. \\ Jin-Joo Park ${ }^{10}$ - Shiro Ishihara ${ }^{11}$ - Khalid F. AlHabib ${ }^{12}$ - Aldo Maggioni ${ }^{13}$ ' Òscar Miró ${ }^{14}$ - Naoki Sato ${ }^{11}$. \\ Alain Cohen-Solal ${ }^{15} \cdot$ Enrique Fairman $^{16} \cdot$ Johan Lassus $^{17} \cdot$ Veli-Pekka Harjola $^{17}$. Christian Mueller $^{18}$. \\ Franck W. Peacock ${ }^{19}$ • Dong-Ju Choi ${ }^{10}$ • Patrick Plaisance ${ }^{20}$ • Jindřich Spinar ${ }^{8,9}$ • Mikhail Kosiborod ${ }^{21}$. \\ Alexandre Mebazaa ${ }^{1,2,3,4}$ Etienne Gayat ${ }^{1,2,3,4}$ - GREAT (Global Research on Acute Conditions Team) Network and \\ INI-CRCT (Investigation Network Initiative-Cardiovascular and Renal Clinical Trialists) network
}

Received: 7 July 2017 / Accepted: 25 October 2017 / Published online: 28 October 2017

(C) Springer-Verlag GmbH Germany 2017

\begin{abstract}
Background The interaction between chronic medications on admission and the association between serum potassium level and outcome in patients with acute heart failure (AHF) are unknown.

Methods Observational intercontinental study of patients admitted with AHF. 15954 patients were included from 12 cohorts in 4 continents. Main outcome was 90 -day mortality. Clinical presentation (medication use, hemodynamics, comorbidities), demographic, echocardiographic, and
\end{abstract}

Miró Òscar: Member of the ICA-SEMES Research Group, Spain.

Mebazaa Alexandre and Gayat Etienne these authors contributed equally to the work.

Electronic supplementary material The online version of this article (doi:10.1007/s00392-017-1173-3) contains supplementary material, which is available to authorized users.

Matthieu Legrand

matthieu.legrand@aphp.fr

1 Department of Anesthesiology and Critical Care and Burn Unit, St-Louis Hospital, Assistance Publique- Hôpitaux de Paris,, Paris, France

2 AP-HP, Groupe Hospitalier Saint-Louis-Lariboisière-Ferna nd-Widal, Paris, France

3 Université Paris Diderot, Sorbonne Paris Cité, Paris, France

4 UMR 942, INSERM, Paris, France

5 Division of Nephrology, Ambroise Paré university hospital, APHP, University of Paris Ouest-Versailles-St-Quentin-en-Yv elines (UVSQ), Boulogne-Billancourt, Paris, France

6 INSERM U1018 Team5, Research Centre in Epidemiology and Population Health (CESP), Univ. Paris-Sud, UVSQ,

Université Paris-Saclay, Villejuif, France biochemical data on admission were recorded prospectively in each cohort, with prospective adjudication of outcomes. Results Positive and negative linear relationships between 90-day mortality and $\mathrm{sK}+$ above $4.5 \mathrm{mmol} / \mathrm{L}$ (hyperkalemia) and below $3.5 \mathrm{mmol} / \mathrm{L}$ (hypo-kalemia) were observed. Hazard ratio for death was 1.46 [1.34-1.58] for hyperkalemia and 1.22 [1.06-1.40] for hypokalemia. In a fully adjusted model, only hyperkalemia remained associated with mortality (HR 1.03 [1.02-1.04] for each $0.1 \mathrm{mmol} / \mathrm{l}$ change of $\mathrm{sK}+$ above $4.5 \mathrm{mmol} / \mathrm{L}$ ). Interaction tests revealed that the association between hyperkalemia and outcome was significantly affected by chronic medications. The association between hyperkalemia and mortality was absent for patients treated with beta blockers and in those with preserved renal function.

Conclusions In patients with AHF, $\mathrm{sK}+>4.5 \mathrm{mmol} / \mathrm{L}$ appears to be associated with 90-day mortality.

7 INSERM, Centre d'Investigations Cliniques Plurithématique 1433, INSERM U1116, Université de Lorraine, CHRU de Nancy, F-CRIN INI-CRCT, Nancy, France

8 Department of Internal Medicine and Cardiology, University Hospital Brno, Brno, Czech Republic

9 Faculty of Medicine, Masaryk University, Brno, Czech Republic

10 Division of Cardiology, Department of Internal Medicine, Cardiovascular Center, Seoul National University Bundang Hospital, Seoul, South Korea

11 Nippon Medical School Musashi-Kosugi Hospital, Kanagawa, Japan

12 Department of Cardiac Sciences, College of Medicine, King Fahad Cardiac Center, King Saud University, Riyadh, Saudi Arabia 
B-blockers have potentially a protective effect in the setting of hyperkalemia.

Keywords Mortality $\cdot$ Potassium $\cdot$ B-blockers $\cdot$ Heart failure $\cdot$ Renal failure

\section{Introduction}

Patients hospitalized for acute heart failure (AHF) have a high risk of death and rehospitalization [1]. Among metabolic disorders, serum potassium level has been a factor of interest in this setting. Serum potassium $(\mathrm{sK}+)$ is under the control of several factors that can be altered in patients with AHF including renal function or activation of renin-angiotensin-aldosterone system [2, 3]. Hypo- and hyperkalemia might, therefore, represent a direct risk of lethal cardiovascular complication but also an indirect biomarker of the severity of the underlying disease (i.e., neurohormonal activation or renal dysfunction) [4]. Several treatments administered to heart failure patients also interact with $\mathrm{sK}+$ (e.g., nonpotassium sparing diuretics, angiotensin-converting enzyme inhibitors or angiotensin receptor blockers, beta blockers or mineralocorticoid receptor antagonists aldosterone) and lead to a risk of hyperkalemia that may curb their prescription [5-9]. Furthermore, very little data are yet available on the interaction between chronic medications and dyskalemia on prognosis [10]. The objective of this study was to describe the association between $\mathrm{sK}+$ at hospital admission and 90-day mortality in patients with AHF, and the interaction of treatment on this association.

\footnotetext{
13 ANMCO Research Center, Firenze, Italy

14 Emergency Department, Hospital Clinic, and 'Emergency care: processes and diseases' Research Group, IDIBAPS, Barcelona, Catalonia, Spain

15 Department of Cardiology, Hôpitaux Universitaire Saint Louis-Lariboisière, Assistance Publique des Hôpitaux de Paris, Paris, France

16 Sociedad Argentina de Cardiologia, Area de Investigacion SAC Azcuenaga, Buenos Aires, Argentina

17 Cardiology, Helsinki University and Helsinki University Hospital, Heart and Lung Center, Helsinki, Finland

18 Department of Cardiology and Cardiovascular Research Institute Basel (CRIB), University Hospital, Basel, Switzerland

19 Baylor College of Medicine, Houston, TX, USA

20 Emergency Department, Hôpitaux Universitaire Saint Louis-Lariboisière, Assistance Publique des Hôpitaux de Paris, Paris, France

21 Saint Luke's Mid America Heart Institute and University of Missouri-Kansas City, Kansas, MO, USA
}

\section{Methods}

The study population was derived from the GREAT registry, an international, multicenter, prospective observational cohort of adult patients with AHF as the main diagnosis at hospital admission. Details on the constitution of the GREAT registry have been previously described in detail $[11,12]$. We defined patients as eligible if they were identified as presenting with AHF and had their potassium level measured on arrival at the emergency department. All patients had AHF according to the European Society of Cardiology guidelines. Both patients with new-onset (i.e., no history) heart failure (HF) and with decompensated chronic HF were included. Small centers (which included $<250$ patients) were not included for the homogeneity of the cohort. The principal investigators for each study submitted hospital patient-level data from admission. 21,865 patients are part of the GREAT network registries from 19 centers in 16 countries of 4 continents. After serial exclusion of patients for outcome and serum $\mathrm{K}+$ on admission missing data and from small recruitment centers, 15,954 patients formed the final cohort (Supplemental Digital Content-Fig. 1). Clinical details of presentation (medication use, hemodynamics, comorbidities), as well as demographic, echocardiographic, and biochemical data on admission were recorded prospectively in each cohort, with prospective adjudication of outcomes. Estimated glomerular filtration rate (eGFR) was calculated by the Modified Diet in Renal Disease formula. Normal range of potassium level across centers were 3.5 (CI 95\% 3.5-3.5) and upper limit of 5.1 (CI 95\% 4.9-5.5) mmol/L. Outcomes were assessed at 90 days by medical chart review at each institution. Local institutional review boards/ethics committees approved all study procedures.

\section{Statistical analysis}

Baseline demographic characteristics were expressed as median and interquartile range or frequency, and were compared using nonparametric techniques for continuous data (e.g., Wilcoxon or Kruskal-Wallis test) or chi-square testing for categorical covariates. The primary outcome of our study was all-cause mortality at 90 days postdischarge. Survival time was calculated from the date of admission until the date of death, or the last follow-up. Survival time was calculated from the date of admission until the date of death or last follow-up. Univariate and multivariate Cox proportional hazards regression models were used to estimate effects for each covariate on mortality. Variables included in the multivariate models for adjustment were gender, age, hyponatremia (defined as natremia at admission $<136 \mathrm{mmol} / \mathrm{L}$ ), hyperglycemia 
(defined as glycemia at admission $>7 \mathrm{mmol} / \mathrm{L}$ ), eGFR (dichotomized around $60 \mathrm{ml} / \mathrm{min} / 1.73 \mathrm{~m}^{2}$ ), systolic and diastolic blood pressure at presentation, diabetes, history of heart failure, coronary artery disease and history of atrial fibrillation. We also took into account the region of origin. These factors were chosen because of their previously published association with outcome in patients with ADHF and their statistical association with mortality in univariate analysis in the present cohort. Proportional hazards assumptions were verified. The log-linearity of the effect of kalemia at admission was assessed in all patients and subgroups of patients for 90-day mortality using restricted cubic spline function. Hyper- and hypokalemia cut-off values were not predefined but prospectively defined as the value of kalemia associated with the lowest mortality $\pm 0.5 \mathrm{mmol} / \mathrm{L}$. Our study was composed of a worldwide sample of patients with AHF; given that patients were included from various centers, a potential cluster effect was taken into account in the models. In addition, several subgroups were pre-defined based on kidney function at admission and on chronic treatment of the patients. Three subgroups of kidney function were defined as eGFR $\geq 60 \mathrm{ml} / \mathrm{min} / 1.73 \mathrm{~m}^{2}$, eGFR $<60 \mathrm{ml} /$ $\mathrm{min} / 1.73 \mathrm{~m}^{2}$ and $\mathrm{eGFR}<30 \mathrm{ml} / \mathrm{min} / 1.73 \mathrm{~m}^{2}$. In addition, several subgroups were pre-defined based on kidney function and on chronic treatment. The chronic treatments considered were RASi (angiotensin-converting enzyme inhibitors, ACE-i; angiotensin receptor blockers, ARB; beta blockers, BB; mineralocorticoid receptor antagonists, MRA) and loop diuretics. Last, the association between kalemia and mortality was assessed separately in patients with preserved left ventricular ejection fraction (LVEF) and in those with altered LVEF.

We adjusted for all covariates specified in the main multivariable Cox regression model for these subgroup analyses. Statistical analyses were performed using R (version 2.15.0, R Foundation for Statistical Computing, Vienna, Austria). A 2 -sided $p$ value $<0.05$ was considered to be statistically significant.

\section{Results}

\section{Study population}

Patients presenting with hyperkalemia, as defined by serum potassium $>4.5 \mathrm{mmol} / \mathrm{L}$, had lower glomerular filtration rate and more frequently had a history of heart failure. They had higher plasma brain natriuretic peptide on admission and hyperglycemia, both prognostic factors of poor outcome in heart failure patients suggesting more advanced heart failure compared to patients admitted with hypo- or normokalemia. Hemodynamics parameters were slightly but significantly different in patients with hyperkalemia with lower systolic and diastolic blood pressure and lower left ventricular ejection fraction. Characteristics of patients are presented in Table 1 .

\section{Association between serum potassium level and 90-day mortality}

Figure 1 shows the linear relationships between 90-day mortality and hyper- (above $4.5 \mathrm{mmol} / \mathrm{L}$ ) and hypokalemia (below $3.5 \mathrm{mmol} / \mathrm{L}$ ). The hazard ratio for death was 1.46 [1.34-1.58] for hyperkalemia and 1.22 [1.06-1.40] for hypokalemia, both versus normokalemia (Fig. 2). However, in a fully adjusted model taking into account potential confounding factors associated with poor outcome (see methods for details) only hyperkalemia remained associated with mortality, 1.03 [1.02-1.04] for $0.1 \mathrm{mmol} / \mathrm{l}$ changes of $\mathrm{sK}+$ above $4.5 \mathrm{mmol} / \mathrm{L}$ and not hypokaliemia (HR 1.03 [0.98-1.09] for $0.1 \mathrm{mmol} / 1$ changes of sK+ below $3.5 \mathrm{mmol} / \mathrm{L}$ ) (Table 2 ).

\section{Influence of LVEF or renal function on the relationship between increased $\mathrm{sK}+$ and short-term outcome}

In univariate analysis, an association between hyperkalemia and mortality exists only in patients with eGFR $<60 \mathrm{ml} / \mathrm{min} / 1.73 \mathrm{~m} 2$. The threshold of the association between potassium level and poor outcome appears to be higher (i.e., $5 \mathrm{mmom} / \mathrm{L}$ ) in patients with severe renal dysfunction (eGFR $<30 \mathrm{ml} / \mathrm{min} / 1.73 \mathrm{~m} 2$ ) (Fig. 3). LVEF had no impact on the incidence of hyperkalemia on association with outcome.

\section{Influence of chronic heart failure medications on the relationship between increased sK+ and outcome}

Patients' treatment rates with ACE-i, ARB, MRA, and BB were no different as compared with normo-, hypo- or hyper kalemia (Table 1). However, patients with hyperkalemia received diuretics and nitrate more often, as would be consistent with more advanced heart failure.

Figure 3 shows that the association between hyperkalemia and mortality remained when acute heart failure patients were treated or not with oral RASi, MRA or loop diuretics on admission, but was not observed when patients were treated with oral BB. Effects of heart failure medications on the association between low $\mathrm{sK}+$ and short-term outcomes are shown in Table 2. Table 2 confirms that, even after adjustment for potential confounding factors, the association between hyperkalemia and short-term mortality remained 
Table 1 Characteristics of patients included in the study,

\begin{tabular}{|c|c|c|c|c|c|}
\hline Variables & All patients & $\begin{array}{l}\text { Normokalemia }(3.5- \\
4.5 \mathrm{mmol} / \mathrm{L})(n=10,013)\end{array}$ & $\begin{array}{l}\text { Hypokalemia } \\
(<3.5 \mathrm{mmol} / \mathrm{L}) \\
(n=1421)\end{array}$ & $\begin{array}{l}\text { Hyperkalemia } \\
(>4.5 \mathrm{mmol} / \mathrm{L}) \\
(n=4520)\end{array}$ & $p$ value \\
\hline Age & $72(61.4$ to 80$)$ & 71.5 (60.6 to 80$)$ & $71.8(60.8$ to 79.9$)$ & $73.1(63.3$ to 80.7$)$ & $<0.0001$ \\
\hline Female gender & $6942(43.5)$ & $4271(42.7)$ & $681(47.9)$ & $1990(44)$ & 0.00063 \\
\hline BMI & $26.3(23.2$ to 30.1$)$ & 26.3 (23.4 to 30$)$ & $26.4(23.2$ to 30.1$)$ & 26.1 (22.9 to 30.2$)$ & 0.47 \\
\hline COPD, n(\%) & $2245(15.2)$ & $1357(14.7)$ & $197(14.5)$ & $691(16.3)$ & 0.041 \\
\hline Hypertension, n(\%) & $10,520(66.6)$ & $6447(65)$ & $963(69)$ & $3110(69.4)$ & $<0.0001$ \\
\hline Previous HF, n(\%) & $7276(46.5)$ & $4417(45)$ & $580(41.5)$ & $2279(51.4)$ & $<0.0001$ \\
\hline $\mathrm{AFib}, \mathrm{n}(\%)$ & $4366(28.1)$ & $2717(27.8)$ & $392(28.6)$ & $1257(28.5)$ & 0.66 \\
\hline $\mathrm{CAD}, \mathrm{n}(\%)$ & $7954(50.2)$ & 4965 (49.9) & $718(50.8)$ & $2271(50.7)$ & 0.63 \\
\hline Diabetes, $n(\%)$ & $6687(42.2)$ & $3970(39.9)$ & $510(36.4)$ & 2207 (49.2) & $<0.0001$ \\
\hline $\mathrm{BB}, n(\%)$ & 6015 (39.7) & $3823(40.1)$ & $541(40.1)$ & $1651(38.4)$ & 0.16 \\
\hline $\mathrm{ACEi}, n(\%)$ & $7283(49.1)$ & $4600(49.4)$ & $621(47.5)$ & $2062(48.9)$ & 0.42 \\
\hline $\mathrm{ARB}, n(\%)$ & $2985(20.1)$ & $1911(20.5)$ & $227(17.4)$ & $847(20.1)$ & 0.03 \\
\hline Diuretics, $n(\%)$ & $7156(58.8)$ & $4419(57.2)$ & $618(58.2)$ & $2119(62.6)$ & $<0.0001$ \\
\hline MRA, $n(\%)$ & $3877(25.9)$ & $2436(25.8)$ & $328(25.3)$ & $1113(26.1)$ & 0.83 \\
\hline Nitrate, $n(\%)$ & $2000(17.4)$ & $1245(17.2)$ & $141(13.7)$ & $614(19)$ & 0.00034 \\
\hline ASA, $n(\%)$ & $4863(43.1)$ & $3022(42.5)$ & $416(41.1)$ & $1425(45)$ & 0.026 \\
\hline Statin, $n(\%)$ & $3716(34.6)$ & $2326(34.4)$ & $318(32.1)$ & $1072(35.9)$ & 0.074 \\
\hline $\mathrm{SBP}(\mathrm{mmHg})$ & $130(110$ to 153$)$ & $132(113-154)$ & $131(110-155)$ & $130(110$ to 150$)$ & $<0.0001$ \\
\hline DBP (mmHg) & 79 (66 to 90$)$ & $80(67-90)$ & $80(67-90)$ & $77(65-90)$ & $<0.0001$ \\
\hline HR (bpm) & $88(73-105)$ & $88(73-105)$ & 89 (75-107) & $86(72-104)$ & 0.00078 \\
\hline $\mathrm{LVEF}<40 \%, n(\%)$ & $4577(42.8)$ & $2881(42.7)$ & $440(43.3)$ & $1256(42.6)$ & 0.93 \\
\hline $\mathrm{Hb}, \mathrm{g} / \mathrm{dl}$ & $12.8(11.2-14.2)$ & $13(11.4-14.3)$ & $12.7(11.2-14.2)$ & $12.3(10.7-14)$ & $<0.0001$ \\
\hline $\mathrm{Na}+(\mathrm{mmol} / \mathrm{L})$ & $138(135-141)$ & $138(135.8-141)$ & $139(135-141)$ & $137(134-140)$ & $<0.0001$ \\
\hline eGFR (ml/min/1.73 m2) & $53.8(38.7-71.1)$ & $57.7(43.1-74.4)$ & $57.6(44.1-74.8)$ & $43.4(29.1-60.1)$ & $<0.0001$ \\
\hline Glucose (mmol/L) & $9.9(6.5-88)$ & $9.4(6.5-80)$ & $10(6.7-62.2)$ & $11.3(6.8-101)$ & $<0.0001$ \\
\hline $\mathrm{BNP}(\mathrm{pg} / \mathrm{mL})$ & $913.5(415.8-1739.2)$ & 840 (389-1637) & 905 (388.8-1697.8) & 1118 (519-2105.3) & $<0.0001$ \\
\hline
\end{tabular}

$p$ values correspond to chi-2 or Kruskal-Wallis test, as appropriate

$B M I$ body mass index, $C O P D$ chronic obstructive pulmonary disease, $G F R$ glomerular filtration rate, $H F r E F$ heart failure with reduced left ventricular ejection fraction, $A f i b$ atrial fibrillation, $C A D$ coronary artery disease, $A C E i$ angiotensin-converting enzyme inhibitors, $A R B$ angiotensin receptor blockers, $B B \mathrm{BBs}, M R A$ mineralocorticoids receptors antagonists, $A S A$ acetylsalicylic acid, $S B P$ systolic blood pressure, $D B P$ diastolic blood pressure, $H R$ heart rate, $L V E F$ left ventricular ejection fraction, $H b$ hemoglobin, $B N P$ B-type natriuretic peptide

significant with all heart failure medications except oral BB on admission.

\section{Discussion}

In this study, we observed that $\mathrm{sK}+$ was associated with 90-day mortality in a U-shaped curve linking mortality and $\mathrm{sK}+$ in acute heart failure patients. However, only hyperkalemia $(>4.5 \mathrm{mmol} / \mathrm{L})$, but not hypokalemia $(<3.5 \mathrm{mmol} / \mathrm{L})$, remained a risk factor of short-term death after adjustment for potential confounding factors. Patients with normal renal function seem not to be exposed to risks of hyperkalemia, while most chronic heart failure medications did not alter the negative association between outcome and high sK + , except in patients on $\mathrm{BB}$ for whom high sK+ was not a risk factor of short-term death.

\section{Association between hypokalemia and outcome}

Low sK+ has long been recognized as a risk of death in heart failure patients. Korgaonkar et al [13], in a cohort of 820 patients with chronic renal failure observed an increased in mortality risk with hypokalemia. In this line, Ahmed et al [10], observed an increased risk of mortality in heart failure patients with low sK+. Interestingly, scales have been proposed for risk stratification (7- and 30-day mortality, respectively) of patients with AHF at emergency departments arrival, including potassium among the variables needed to calculate the score in a very similar way that 
Fig. 1 Distribution of serum potassium level on admission (a) and relationship between potassium serum level on admission and probability of death at 90 days. Results presented in median (IC 95\%). The U-shape curve indicated an increased risk of death associated with both hypo- and hyperkalemia with a narrow range of $\mathrm{K}+\operatorname{serum}(3.5-4.5 \mathrm{mmol} / \mathrm{L})$ centered on $\mathrm{sK}+=4 \mathrm{mmol} / \mathrm{L}$ associated with the lowest risk of death (b)
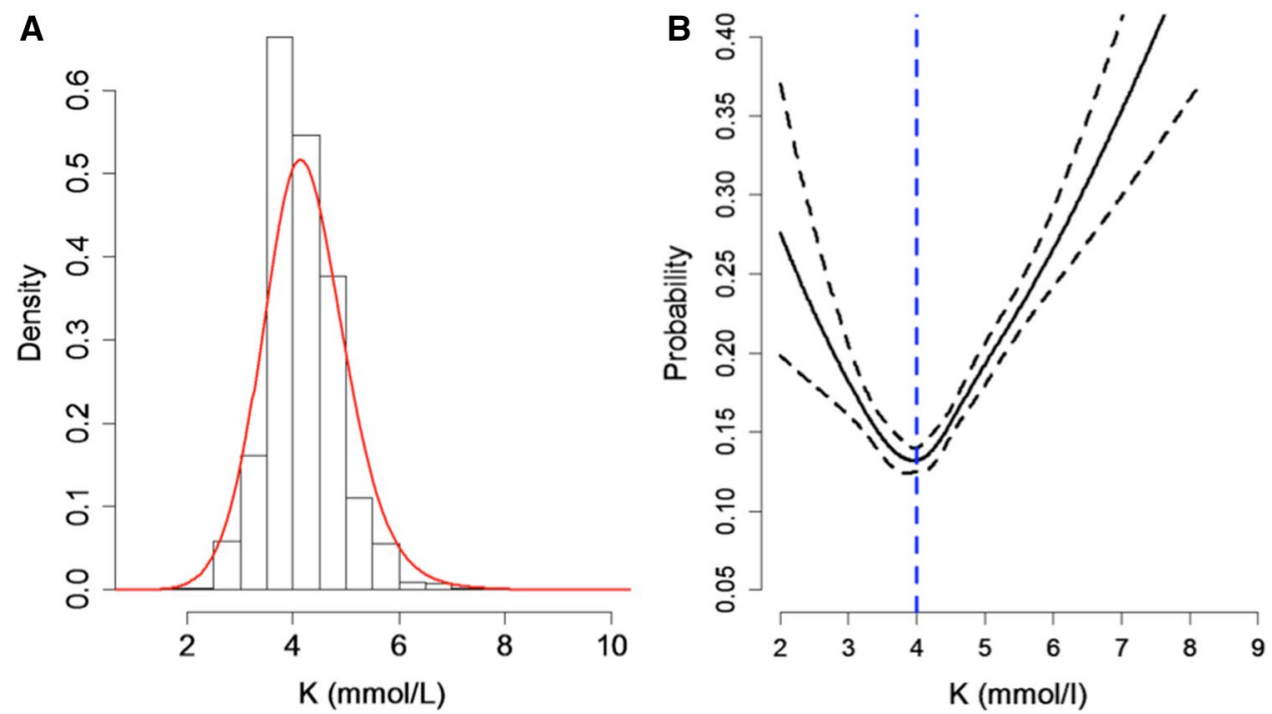

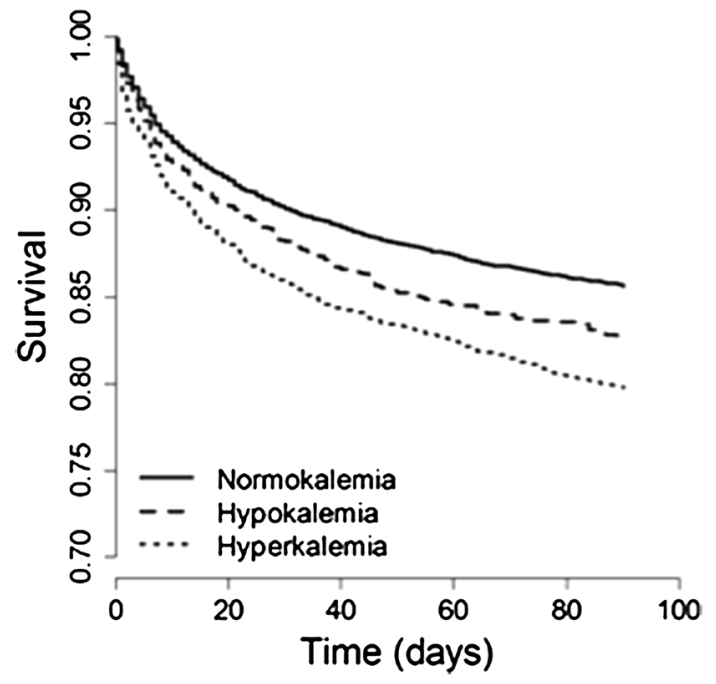

Fig. 2 Kaplan-meier curves showing survival curves in patients with hypokalemia $(\mathrm{K}+<3.5 \mathrm{mmol} / \mathrm{L})$, normokalemia $(\mathrm{K}+3.5-$ $4.5 \mathrm{mmol} / \mathrm{L})$ and hyperkalemia $(\mathrm{K}+>4.5 \mathrm{mmol} / \mathrm{L})$

we have found in the present study: both confer a worse prognosis, with hyperkalemia having a most detrimental impact on it [14]. In addition, association between sK+ $<4 \mathrm{mmol} / \mathrm{L}$ and prognosis was found in two large cohorts of chronic heart failure patients with chronic renal failure after matching with a propensity score $[15,16]$, suggesting that hypokalemia could be a marker of severity of heart failure rather than a causal factor of death. This assumption is reinforced by the fact that potassium-sparing treatments such MRA may improve survival, unlike potassium supplementation [5, 17]. Others, however, raised concerns regarding risk of arrhythmia, sudden deaths and vascular effects with hypokalemia [18]. The non-significant association between hypokalemia and mortality after adjustment for confounding factors in our study also strongly suggested that hypokalemia may represent a marker of disease severity rather than an independent factor of poor outcome in heart failure.

\section{Association between hyperkalemia and outcome}

Less common are studies linking hyperkalemia and the risk of death in heart failure patients. In our study, we observed that the $\mathrm{sK}+$ threshold for poor outcome is lower than usually proposed as a definition of hyperkalemia. The risk of dying increased by $3 \%$ per $0.1 \mathrm{mmol} / \mathrm{L}$ increase in serum potassium level which appears clinically significant. This suggests that definition for hyperkalemia with $\mathrm{sK}+>5-5.5 \mathrm{mmol} / \mathrm{L}$ might be too restrictive [18]. Ahmed et al. have demonstrated an increased risk of death with $\mathrm{sK}+>5-5.5 \mathrm{mmol} / \mathrm{L}$ in a cohort of chronic heart failure after adjustment with a propensity score analysis [19]. Altered renal function at admission appears to affect the relationship between high $\mathrm{sK}+$ and mortality in 2 ways. First, the relationship between high $\mathrm{sK}+$ and increased short-term mortality was only seen in acute heart failure patients with impaired renal function (eGFR $<60 \mathrm{ml} / \mathrm{min} / 1.73 \mathrm{~m} 2)$. Second, the presence of severe renal impairment (eGFR $<30 \mathrm{ml} /$ $\min / 1.73 \mathrm{~m} 2$ ) appeared to right shift the slope of the association between $\mathrm{sK}+$ and mortality (about $0.5 \mathrm{mmol} / \mathrm{L}$ ). Those findings could be related to hyperkalemia tolerance when $\mathrm{sK}+$ is chronically elevated. The association between high $\mathrm{sK}+$ and mortality remained after adjustment for potential confounding factors suggesting that serum potassium greater than $4.5 \mathrm{mmol} / \mathrm{L}$ is a risk factor of short-term death in acute heart failure. 
Table 2 Hazard ratio of hyper and hypokalemia for 90-day mortality

\begin{tabular}{|c|c|c|c|c|c|}
\hline Sub-groups & $\begin{array}{l}\text { "Normal" range of } \\
\text { kalemia }\end{array}$ & $\begin{array}{l}\text { OR for each } 0.1 \mathrm{mmol} / \mathrm{l} \\
\text { below the lower limit }\end{array}$ & $p_{\text {interaction }}$ & $\begin{array}{l}\text { OR for each } 0.1 \mathrm{mmol} / 1 \\
\text { above the upper limit }\end{array}$ & $p_{\text {interaction }}$ \\
\hline All patients $(n=15954)$ & $3.5-4.5$ & $1.03[0.98-1.09]$ & - & $1.03[1.02-1.04]^{*}$ & - \\
\hline $\mathrm{eGFR}>=60(n=5332)$ & $3.3-4.3$ & $1.09[1.03-1.15]$ & 0.005 & 1.02 [0.99-1.04] & 0.04 \\
\hline eGFR $<60(n=7893)$ & $3.5-4.5$ & $1.03[0.97-1.09]$ & & $1.03[1.02-1.04]^{*}$ & \\
\hline eGFR $<30(n=1858)$ & $4.0-5.0$ & $1.05[1.03-1.07]^{*}$ & & $1.03[1.01-1.05]^{*}$ & \\
\hline $\operatorname{HFrEF}(n=4577)$ & $3.2-4.2$ & $1.14[1.08-1.21]$ & 0.01 & $1.02[1.01-1.03]^{*}$ & 0.04 \\
\hline $\operatorname{HFpEF}(n=8128)$ & $3.55-4.55$ & $1.02[0.96-1.08]$ & & $1.02[1.01-1.03]^{*}$ & \\
\hline No RASi $(n=5837)$ & $3.5-4.5$ & $1.05[0.99-1.12]$ & $<0.0001$ & $1.02[1.00-1.04]^{*}$ & 0.005 \\
\hline RASi $(n=8995)$ & $3.5-4.5$ & 0.98 [0.94-1.02] & & $1.03[1.02-1.04]^{*}$ & \\
\hline No BB $(n=9154)$ & $3.4-4.5$ & $1.08[1.01-1.16]^{*}$ & 0.04 & $1.03[1.02-1.04]^{*}$ & 0.009 \\
\hline $\mathrm{BB}(n=6015)$ & $3.6-4.6$ & $0.99[0.92-1.06]$ & & 1.01 [0.95-1.08] & \\
\hline No diuretic $(n=5020)$ & $3.3-4.3$ & $1.22[1.07-1.38]^{*}$ & 0.001 & $1.03[1.01-1.05]^{*}$ & $<0.0001$ \\
\hline Diuretic $(n=7156)$ & $3.5-4.5$ & $1.05[1.02-1.08]^{*}$ & & $1.04[1.03-1.05]^{*}$ & \\
\hline No MRA $(n=11,102)$ & $3.5-4.5$ & 1.03 [0.99-1.07] & 0.91 & $1.03[1.02-1.05]^{*}$ & $<0.0001$ \\
\hline MRA $(\mathrm{n}=3877)$ & $3.5-4.5$ & $1.02[0.91-1.13]$ & & $1.01[1.00-1.03]^{*}$ & \\
\hline
\end{tabular}

Statistically significant values are appear in bold

Hazard ratio of hyper and hypokalemia for 90 days mortality after adjustment for potential confounding factors (see text for details) according to estimated glomerular filtration rate (eGFR), heart failure status ( $H F r E F$ Heart failure with reduced ejection fraction, $H R p E F$ heart failure with preserved ejection fraction) and treatments on admission ( $R A S i$ renin-angiotensin system inhibitors, MRA mineralocorticoids receptors antagonists). Note than thresholds for hyper and hypokalemia were adjusted for each condition based on the relationship between sK+, GFR and treatments (Fig. 3)

$*<0.05$

\section{Impact of chronic medications on the association between serum potassium level and outcome}

Significant information provided by our study is the influence of chronic heart failure therapies, most of which are known to affect $\mathrm{sK}+$, on the relationship between mortality and sK + . Several authors have shown that chronic treatment by MRA is associated with a moderate increase in $\mathrm{sK}+$ in heart failure patients $[2,20]$. Likewise, chronic administration of ACE inhibitors or ARB was already described as a risk factor of hyperkalemia in heart failure patients. The consequences of a rise of $\mathrm{sK}+$ on the prognosis of patients with acute heart failure, however, have not been thoroughly explored.

In our study, taking into account potential confounding factors associated with poor outcome, only hyperkalemia remained associated with mortality in patients with altered eGFR. Our study also showed that though no causal relationship can be drawn from this observational data, treatment with BB might counteract the detrimental effect of dyskalemia on outcome, suggesting a protective effect of BB toward hyperkalemia. This might, at least partly explain, the striking benefits of $\mathrm{BB}$ on short-term outcome in AHF patients previously described $[21,22]$. Our study has several limitations. First, we cannot exclude pseudo-hyperkalemia in some patients. However, the size of the cohort reduces the risk of error when interpreting the results in reducing this noise. Only renal function and $\mathrm{sK}+$ values at hospital admission were available and the values prior to admission were unknown. Also, the mechanism of hyperkalemia (i.e., transfer or renal excretion deficit) could not be explored. It is possible that mechanisms influence the prognosis of hyperkalemia. We acknowledge that adhesion to guidelines regarding treatment of heart failure was low, especially regarding the use of B-blockers (only $40 \%$ of patients were treated with B-blockers) [23]. This observation was similar to recently published low adherence to guidelines in heart failure patients [24-26]. Potassium intake was not available in this registry. Finally, therapeutic strategies implemented during hospitalization and changes in serum potassium during the hospital stay were not known. However, it should be noted that Khan et al. studied the impact on prognosis relative to changes in serum potassium during hospitalization among AHF patients from the EVEREST trial, and did not detect any impact on mortality [20].

To conclude, after adjustment we observed an association between admission hyperkalemia $>4.5 \mathrm{mmol} / \mathrm{L}$ and 90 day mortality in AHF patients, suggesting that current definitions of hyperkalemia might be too restrictive. This association was not observed in patients treated with B-Blockers, even after adjustment on potential 
Fig. 3 Graphical representation of the relationship between serum potassium level and risk of death according to renal function at admission (a), diuretics (b), RASi (c), left ventricular ejection fraction (d), mineralocorticoid receptors antagonists (e) and B-blockers (f). $e G F R$ estimated glomerular filtration rate, $H F r E F$ heart failure with reduced left ventricular ejection fraction, $H F p E F$ heart failure with preserved left ventricular ejection fraction, $R A S i$ renin angiotensin aldosterone system inhibitors (i.e., angiotensin-converting enzyme inhibitors and angiotensin receptor blockers); $B B$ BBs, $M R A$ mineralocorticoid receptor antagonists
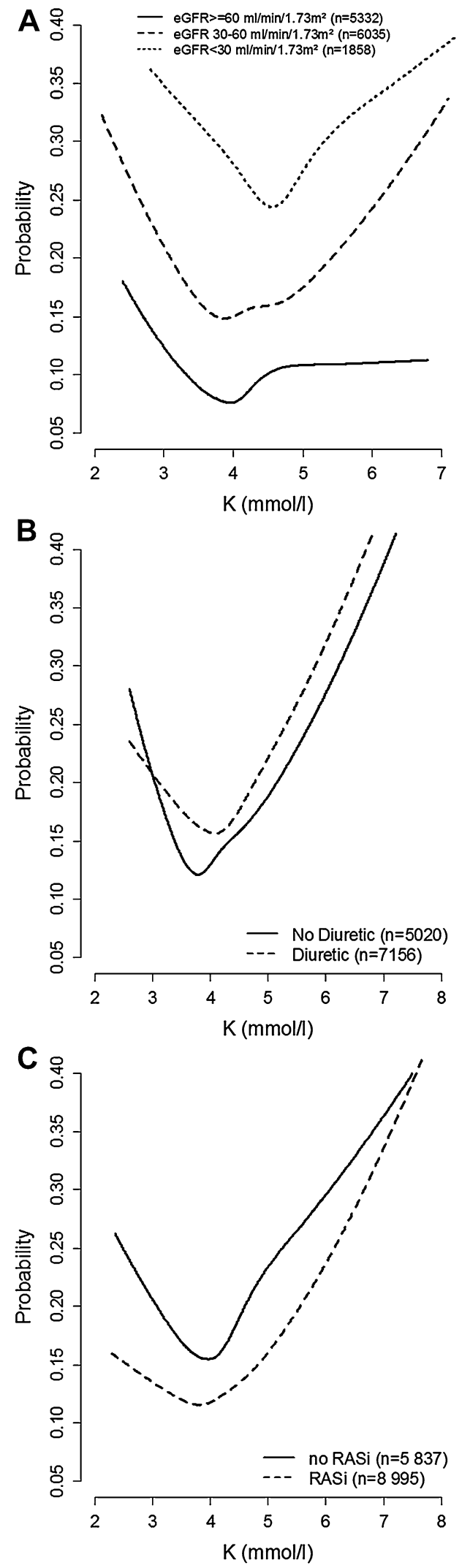
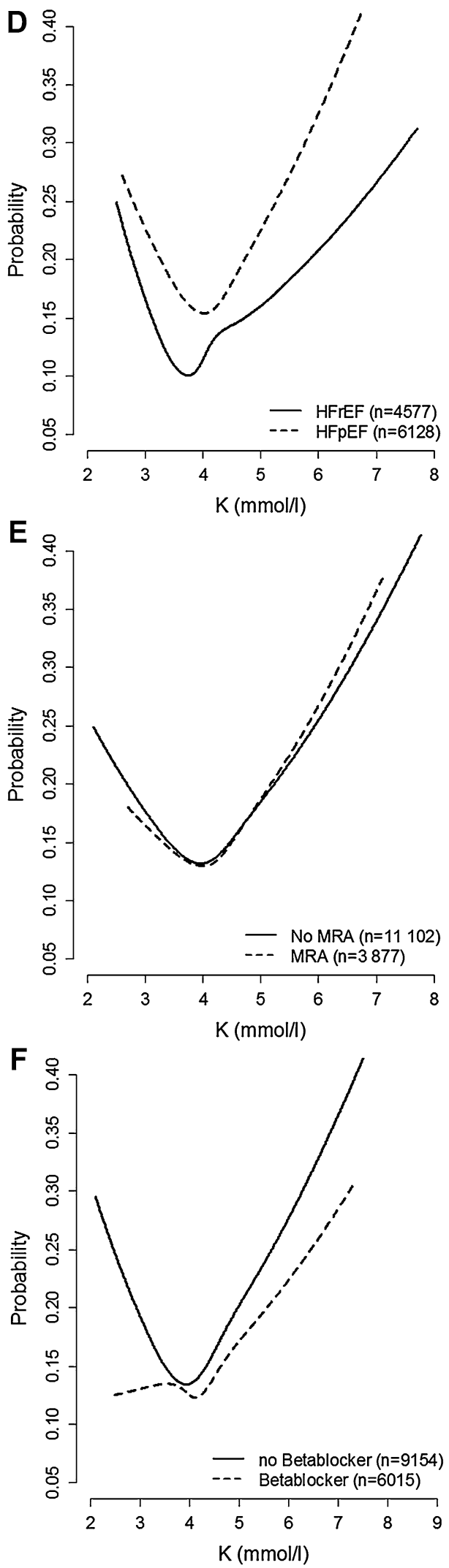
confounding factors. B-blockers may have potentially a protective effect in the setting of hyperkalemia.

Acknowledgements Saudi Heart Association, The Deanship of Scientific Research at King Saud University, Riyadh, Saudi Arabia (Research group number: RG -1436-013).

\section{Compliance with ethical standards}

Funding None.

\section{References}

1. Legrand M, Mebazaa A, Ronco C, Januzzi JL (2014) When cardiac failure, kidney dysfunction, and kidney injury intersect in acute conditions: the case of cardiorenal syndrome. Crit Care Med 42:2109-2117. doi: 10.1097/CCM.0000000000000404

2. Rossignol P, Zannad F, Pitt B, Writing group of 10th Global Cardio Vascular Clinical Trialist forum held on December 6th-7th 2013 in Paris, France (2014) Time to retrieve the best benefits from renin angiotensin aldosterone system (RAAS) inhibition in heart failure patients with reduced ejection fraction: lessons from randomized controlled trials and registries. Int J Cardiol 177:731-733. doi: 10.1016/j.ijcard.2014.11.004

3. Ferreira JP, Girerd N, Medeiros PB et al (2016) Spot urine sodium excretion as prognostic marker in acutely decompensated heart failure: the spironolactone effect. Clin Res Cardiol Off J Ger Card Soc 105:489-507. doi: 10.1007/s00392-015-0945-x

4. Giebisch G, Krapf R, Wagner C (2007) Renal and extrarenal regulation of potassium. Kidney Int 72:397-410. doi: 10.1038/ sj.ki.5002288

5. Eschalier R, McMurray JJV, Swedberg K et al (2013) Safety and efficacy of eplerenone in patients at high risk for hyperkalemia and/or worsening renal function: analyses of the EMPHASIS-HF study subgroups (Eplerenone in mild patients hospitalization and survival study in heart failure). J Am Coll Cardiol 62:1585-1593. doi: 10.1016/j.jacc.2013.04.086

6. Pitt B, Pfeffer MA, Assmann SF et al (2014) Spironolactone for heart failure with preserved ejection fraction. N Engl J Med 370:1383-1392. doi: 10.1056/NEJMoa1313731

7. Albert NM, Yancy CW, Liang L et al (2009) Use of aldosterone antagonists in heart failure. JAMA 302:1658-1665. doi: 10.1001/ jama.2009.1493

8. Fröhlich H, Torres L, Täger T et al (2017) Bisoprolol compared with carvedilol and metoprolol succinate in the treatment of patients with chronic heart failure. Clin Res Cardiol Off J Ger Card Soc. doi: 10.1007/s00392-017-1115-0

9. Matsue Y, Ter Maaten JM, Suzuki M et al (2017) Early treatment with tolvaptan improves diuretic response in acute heart failure with renal dysfunction. Clin Res Cardiol Off J Ger Card Soc. doi: 10.1007/s00392-017-1122-1

10. Ahmed A, Zannad F, Love TE et al (2007) A propensity-matched study of the association of low serum potassium levels and mortality in chronic heart failure. Eur Heart J 28:1334-1343. doi: 10.1093/eurheartj/ehm091

11. Arrigo M, Gayat E, Parenica J et al (2017) Precipitating factors and 90-day outcome of acute heart failure: a report from the intercontinental GREAT registry. Eur J Heart Fail 19:201-208. doi: 10.1002/ejhf.682

12. Mebazaa A, Gayat E, Lassus J et al (2013) Association between elevated blood glucose and outcome in acute heart failure: results from an international observational cohort. J Am Coll Cardiol 61:820-829. doi: 10.1016/j.jacc.2012.11.054

13. Korgaonkar S, Tilea A, Gillespie BW et al (2010) Serum potassium and outcomes in CKD: insights from the RRI-CKD cohort study. Clin J Am Soc Nephrol CJASN 5:762-769. doi: 10.2215/ CJN.05850809

14. Lee DS, Stitt A, Austin PC et al (2012) Prediction of heart failure mortality in emergent care: a cohort study. Ann Intern Med 156:767-775. doi: 10.7326/0003-4819-156-11-201206050-00003 W-261, W-262.

15. Alper AB, Campbell RC, Anker SD et al (2009) A propensitymatched study of low serum potassium and mortality in older adults with chronic heart failure. Int J Cardiol 137:1-8. doi: 10.1016/j.ijcard.2008.05.047

16. Bowling CB, Pitt B, Ahmed MI et al (2010) Hypokalemia and outcomes in patients with chronic heart failure and chronic kidney disease: findings from propensity-matched studies. Circ Heart Fail 3:253-260. doi: 10.1161/CIRCHEARTFAILURE.109.899526

17. Zannad F, Gattis Stough W, Rossignol P et al (2012) Mineralocorticoid receptor antagonists for heart failure with reduced ejection fraction: integrating evidence into clinical practice. Eur Heart J 33:2782-2795. doi: 10.1093/eurheartj/ehs257

18. Macdonald JE, Struthers AD (2004) What is the optimal serum potassium level in cardiovascular patients? J Am Coll Cardiol 43:155-161

19. Ahmed MI, Ekundayo OJ, Mujib M et al (2010) Mild hyperkalemia and outcomes in chronic heart failure: a propensity matched study. Int J Cardiol 144:383-388. doi: 10.1016/j. ijcard.2009.04.041

20. Khan SS, Campia U, Chioncel O et al (2015) Changes in serum potassium levels during hospitalization in patients with worsening heart failure and reduced ejection fraction (from the EVEREST trial). Am J Cardiol 115:790-796. doi: 10.1016/j. amjcard.2014.12.045

21. Böhm M, Link A, Cai D et al (2011) Beneficial association of $\beta$-blocker therapy on recovery from severe acute heart failure treatment: data from the Survival of Patients With Acute Heart Failure in Need of Intravenous Inotropic Support trial. Crit Care Med 39:940-944. doi: 10.1097/CCM.0b013e31820a91ed

22. Jondeau G, Neuder Y, Eicher J-C et al (2009) B-CONVINCED: Beta-blocker CONtinuation Vs. INterruption in patients with Congestive heart failure hospitalizED for a decompensation episode. Eur Heart J 30:2186-2192. doi: 10.1093/eurheartj/ehp323

23. Ponikowski P, Voors AA, Anker SD et al (2016) 2016 ESC Guidelines for the diagnosis and treatment of acute and chronic heart failure: The Task Force for the diagnosis and treatment of acute and chronic heart failure of the European Society of Cardiology (ESC). Developed with the special contribution of the Heart Failure Association (HFA) of the ESC. Eur J Heart Fail 18:891-975. doi: 10.1002/ejhf.592

24. Fu M, Ahrenmark U, Berglund S et al (2017) Adherence to optimal heart rate control in heart failure with reduced ejection fraction: insight from a survey of heart rate in heart failure in Sweden (HR-HF study). Clin Res Cardiol Off J Ger Card Soc. doi: 10.1007/s00392-017-1146-6

25. Hirt MN, Muttardi A, Helms TM et al (2016) General practitioners' adherence to chronic heart failure guidelines regarding medication: the GP-HF study. Clin Res Cardiol Off J Ger Card Soc 105:441-450. doi: 10.1007/s00392-015-0939-8

26. Störk S, Handrock R, Jacob J et al (2017) Treatment of chronic heart failure in Germany: a retrospective database study. Clin Res Cardiol Off J Ger Card Soc. doi: 10.1007/s00392-017-1138-6 